Der Buchverlag 


\section{Eduard Schönstedt}

\section{Der Buchverlag}

Geschichte, Aufbau,

Wirtschaftsprinzipien,

Kalkulation und Marketing

\section{J. B. Metzlersche Verlagsbuchhandlung Stuttgart}


CIP-Titelaufnahme der Deutschen Bibliothek

Schönstedt, Eduard:

Der Buchverlag : Geschichte, Aufbau, Wirtschaftsprinzipien,

Kalkulation und Marketing / Eduard Schönstedt. - Stuttgart :

Metzler, 1991

ISBN 978-3-476-00741-4

ISBN 978-3-476-00741-4

ISBN 978-3-476-03345-1 (eBook)

DOI 10.1007/978-3-476-03345-1

Dieses Werk einschließlich aller seiner Teile ist urheberrechtlich geschützt. Jede Verwertung außerhalb der engen Grenzen des Urheberrechtsgesetzes ist ohne Zustimmung des Verlages unzulässig und strafbar. Das gilt insbesondere für Vervielfältigungen, Übersetzungen, Mikroverfilmungen und die Einspeicherung und Verarbeitung in elektronischen Systemen.

(C) 1991 Springer-Verlag GmbH Deutschland

Ursprünglich erschienen bei J.B. Metzlersche Verlagsbuchhandlung und Carl Ernst Poeschel Verlag GmbH in Stuttgart 1991 
Der Verleger schielt mit einem Auge nach dem Schriftsteller, mit dem anderen nach dem Publikum. Aber das dritte Auge, das Auge der Weisheit, blickt unbeirrt ins Portemonnaie.

Alfred Döblin 


\section{Inhaltsverzeichnis}

Vorwort XI

\section{Teil A: \\ Verlags-Historie}

1. Vor-Geschichte:

Relativierung heutiger Grundvorstellungen 3

1. »Verlag« 4

2. »Buch « 6

3. »Vervielfältigungsstück«, » Kopie«, »Auflage« 9

4. »Druck« 10

2. Entstehungs-Geschichte:

Der Weg zum »reinen « Verlag 15

1. Handschriften-Verleger

2. Drucker-Verleger

3. Sortimenter-Verleger 21

4. Der »reine« Verlag 23

3. Gegenwarts-Geschichte:

Die jüngsten Verlagssysteme im politischen Wechsel 27

1. Das » Leipziger System « 27

2. Die Lizenz-Zeit unter den Alliierten — 30

3. Das Verlagssystem der DDR _ 34

4. Das Verlagssystem der BRD _ 38

4. Trends für die Zukunft:

Die Verlagswirtschaft im Umbruch 43

1. Strukturwandel 43

2. Gemeinsames Deutschland 46

3. Europäischer Binnenmarkt 50 


\section{Teil B:}

Verlagsformen und Wirtschaftsprinzipien

1. Einzelwirtschaftliche Formen 60

1. Selbst- oder Eigenverlag 60

2. Selbstkosten-, Herstellkosten-, Druckkosten(zuschuß)-, »Privat «verlage 62

3. Autoren- und Autoren-Selbstverlage — 67

4. Kollektivverlage oder Verlage in Selbstverwaltung __ 70

5. Universitätsverlage _ 71

6. Kommissionsverlag und Verlag subventionierter Literatur _ 74

7. Gesponsorte Literatur und scheinbar gesponsorte __ 80

8. Verlage als » Regiebetriebe « _ 86

2. Überbetriebliche Kooperationen 90

1. Lizenzhandel und Imprintgeschäft _ـ 90

2. Arbeitsgemeinschaften

3. Koproduktionsgemeinschaft und Joint Ventures __ 95

4. Gemeinschaftsverlage — 97

3. Die Buchgemeinschaften — 101

Teil C:

Aufbau und Organisation im Buchverlag

1. Führungsebene

2. Das Lektorat

3. Die Herstellung

4. Die Werbung

5. Der Vertrieb

1. Die Innenaufgaben

2. Die Auslieferung

3. Der Außendienst

Teil D:

\section{Die Kalkulation von Büchern}

1. Die Kalkulationsmodelle

1. Die »Historische Drittelkalkulation « 
2. Die »Multiplikator-Methode«

3. Die traditionellen Staffel-Kalkulationen

4. Die Berechnung von Deckungsauflagen

5. Das Problem der Gemeinkosten

6. Der »Ausweg « oder die moderne Deckungsbeitragsrechnung

7. Die modernen Deckungsauflagen

2. Kurze Bilanz zur Verlagskalkulation

\section{Teil E: \\ Marketing für Buchverlage}

I. Das absatzpolitische Instrumentarium __ 163

1. Distributionspolitik — 163

1. »Eigen- oder Fremdauslieferung? « 164

2. »Reisende oder Verlagsvertreter? « 166

3. Die Wahl der Vertriebskanäle _ـ 172

2. Kommunikationspolitik — 182

1. Die »klassische Werbung « _ 183

2. Öffentlichkeitsarbeit (PR) 191

3. Verkaufsförderung — 194

3. Preispolitik 200

1. Ladenpreispolitik — 200

2. Rabattpolitik 205

3. Konditionenpolitik — 209

4. Produktpolitik — 211

1. Innovation neuer Produkte _ـ 212

2. Gestaltung neuer Produkte _ 213

3. Einführung neuer Produkte 219

4. Produktpolitische Strategien _ 223

II. Das beschaffungspolitische Instrumentarium — 229

1. Marktforschung für Buchverlage _- 229

1. Professionelle oder gewerbliche Marktforschung _ 229

2. Marktbeobachtung oder » Marketing Intelligence «_ 233 
2. Kreation neuer Ideen oder »Ideenmarketing " _ 234

1. Quellen für neue Ideen

234

2. Methoden für neue Ideen oder Kreativtechniken im Verlag 236

3. »Autorenmarketing« 242

\section{Teil F: \\ Anhang}

Bibliographie 249

Anmerkungen 261

Nachweise der Motti 264

Fachadressen für die Aus- und Weiterbildung 265

Stichwort- und Namens-Register 267 


\section{Vorwort}

»Die Buchhändler sind alle des Teufels, für sie muß es eine eigene Hölle geben." Keinem geringeren als Johann Wolfgang von Goethe entfuhr dies als Fluch in einem Gespräch im Jahre 1829, aber gemünzt war die zitierwürdige Äußerung nicht auf die Sortimenter, sondern auf Johann Friedrich Cotta, seinen Verleger.

Verteufelt werden die Verleger in diesem Buch nicht, auch wenn es seit Goethe nicht aufgehört hat, daß Autoren, Buchhändler und Leser Verleger verteufeln: Bei Goethe speziell war der Grund Cottas Zaudern bei der Herausgabe der Goetheschen Werke, bei Autoren schlechthin ist es (zum Beispiel) notorisch der zu geringe Einsatz des Verlags für die Werbung, beim Buchhändler der Rabatt, der zu niedrig, und beim Leser der Ladenpreis, der $\mathrm{zu}$ hoch ist. Unter Insidern haben Verleger zuweilen sogar ein Image, das nachträglich die Gestalten der Bibel verändert. Wie schreibt doch Lord Byron an einschlägiger Stelle? »Barabbas aber war ein Verleger.«

Genau dem ökonomischen, kommerziellen, nicht dem geistig-ideellen Aspekt, sondern der »Ware « Buch ist dieses Buch gewidmet - genauso, wie es Döblin in seinem Bonmot formuliert hat. Es wird gezeigt, wie Verleger wirtschaftlich handeln müssen, um ihre kulturelle Aufgabe erfüllen zu können. Der Leser wird dabei nicht nur erfahren, wie es zur Entwicklung der Verlage in unserer heutigen Form kommen mußte, sondern auch, was der moderne Verlag von der Akquisition der Autoren bis zu Durchführung des Absatzes konkret zu tun hat. Produktionsrisiken, Buchhandelsrabatte, Ladenpreise wie aber auch Wirtschaftsphilosophie oder Marketing der Verlage werden dadurch verständlich.

Die Branche, um die es hier geht, ist einerseits von beeindruckender Größe. Etwa 2000 Buchverlage sind Mitglied im Börsenverein. Etwa 3000 Verlage kommen hinzu, wenn man auf Presseverlage erweitert. Geht man wieder auf die Buchbranche zurück, stehen neben den Mitgliedsverlagen über 3500 Mitgliedsbuchhandlungen. Zählt man auch diejenigen Verlage von Büchern, die nicht Mitglied im Börsenverein sind, entstehen nach dem Verzeichnis lieferbarer Bücher (VLB) über 8500 Firmen, nach dem Adreßbuch für den deutschsprachigen Buchhandel kommen über 12000 zusammen und die Gesamtheit aller Händler im herstellenden und verbreitenden Buchhandel bringt es inklusive Zwischenbuchhandel auf 17200 Adressen. Die Buchtitelneuproduktion belief sich zur Buchmesse 1990, das noch nicht einmal ein 
Titelrekordjahr darstellte, auf 62500, was auf den Arbeitstag umgerechnet über 200 Titel täglich neu bedeutet. Neben diesen neuen stehen über 500000 , die nach dem VLB lieferbar sind, obwohl längst nicht alle Verlage ihre Titel dort sigeln. Die Umsätze mit Büchern belaufen sich (1990 für das Vorjahr erhoben) mit den Fachzeitschriften der Buchverlage auf zwölf Milliarden Mark auf allen Vertriebswegen, nimmt man die Verlage der Presse wieder hinzu, entstehen zusätzliche 36 Milliarden oder 48 total. Und genauso beeindruckend ist der Blick auf die Beschäftigtenzahlen, insbesondere im verlagsnahen Branchenvergleich. Für die Verlage arbeiteten im Erhebungszeitraum für Buch und Presse in allen Arbeitstätten zusammen über 270000 Beschäftigte - für die Druckindustrie waren es 180000 , die etwa 33 Milliarden umsetzten und in der Werbewirtschaft knapp 100000 mit etwa 24 Milliarden Mark Jahresumsatz.

Die beeindruckende Bilanz der Verlagswirtschaft ändert sich aber bzw. wird erst dann einschätzbar, wenn man sie mit »fremden « Branchen oder der Gesamtwirtschaft in Vergleich setzt. Der Handel mit Büchern beträgt dann, gemessen am Gesamteinzelhandel (mit Lebensmitteln, Textilien, Radio und TV etc.) gerade ein Hundertstel, und der Anteil am Bruttosozialprodukt (2260 Milliarden) beträgt für die Buchverlage 0,53 Prozent. In absoluten Zahlen entspricht das dem Umsatz eines einzigen Kaufhauskonzerns oder den Kontoführungsgebühren, welche die Banken in der BRD gegenüber ihren Kunden erheben.

Innerhalb dieses einerseits großen, andererseits minimalen wirtschaftlichen Rahmens erscheint »Der Buchverlag « zu einem für die Entwicklung des Verlagswesens brisanten Zeitpunkt. Der "Strukturwandel « liegt (aber bis zu welchem Teil?) hinter uns, das »Gemeinsame Deutschland « (mit seinen Chancen) konstituiert sich, der »EG-Binnenmarkt « (mit seinen Unsicherheiten und sicher Gefahren) ab 1993 liegt vor uns. Aber nicht nur die wirtschaftlichen Umbrüche werden die Verlagswirtschaft ändern, sondern auch die technologischen, die gerade nach 1990 auch für das Buch zu neuen Medien führen. Vom gedruckten Produkt, der Grundlage für die Buchverlagswirtschaft, hieß es schon vor zehn Jahren, daß es in Zukunft als buchgroßer Körper mit Flüssigkristallbildschirm erscheine, in dem man, je nach Bedarf, literarische, wissenschaftliche und sogar farbbildfähige ganze Verlagsprogramme/Programmbibliotheken einspeisen könne. Auf der Buchmesse 1990 haben - nach Mikrofiches, PC-Kassetten, Disks und CD-ROMs europäischer und amerikanischer Provenienz - Japaner ihren nur postkartengroßen »Elektronic Book Player « von erstaunlicher Speicherkapazität und mit, für ein Druckprodukt, unerreichbarem Benutzerkomfort vorgestellt, dies "Buch « steht also vielleicht bald neben den anderen Formen, die wir bereits aus dem Electronic Publishing kennen. »Der Buchverlag« versucht zusammenzufassen, was sich im Bereich des gedruckten Produkts tun läßt, aber manches wäre schon übertragbar. 
Ein paar Bemerkungen sind noch für das Lesen des »Buchverlags « nötig. Er beginnt wie ein Lesebuch, wird dann zum Sachbuch, und ist am Ende beinahe ein Lehrbuch. Er greift dabei auf über sechs Jahre eigener praktischer Tätigkeit in Verlagen und nahezu zehn in Lehre und teilweiser Forschung zurück. Er verarbeitet ferner Gespräche mit Verlegern, Autoren, Herstellern, Vertriebs- und Werbespezialisten, dazu die auf Branchenseminaren, Verlagsexkursionen und Buchmessen des In- und Auslandes gewonnenen Informationen, Sendungen aus Hörfunk und Fernsehen und Zufallsfunde aus Zeitungen und Zeitschriften - neben natürlich "Börsenblatt «, "Buchmarkt «, »Buchreport « sowie der » eigentlichen « Literatur. Für den Leser ergibt sich vor diesem Hintergrund positiv, daß dies Buch fast ohne Anmerkungen auskommen muß, nur das Notwendigste (wie zentrale Zitate) wird nachgewiesen. Die Leserschaft für das Buch müßte vielfältig sein. An den Universitäten ermöglicht es allen mit der Materie Buch besonders verbundenen Studierenden einen Einblick in den verlegerischen bzw. kaufmännischen Literaturbetrieb. Im Ausbildungswesen für den Buchhandel fundiert oder erweitert es für Verlagsbuchhändler, Verlagskaufleute, Sortimenter und Bibliothekare die Fachausbildung. In Verlagen kann es Lektoren, Herstellern, Werbern, Vertriebs- und Marketingkräften, Kalkulatoren und Verlegern zum Nachschlagen darüber dienen, was gegenüber den im eigenen Verlag realisierten Modellen anders sein könnte. Für direkt das Buch- oder Verlagswesen Studierende dürfte es ein Baustein im Rahmen des Fachstudiums sein, Autoren (nicht nur potentielle) dürften als Leser in Frage kommen, sowie der von den Buchhändlern und Verlegern seit Gutenberg gesuchte »am Buch allgemein Interessierte«. Fachlesern sei aber vorab erklärt, daß sie nicht immer das ihnen Vertraute vorfinden. Lücken mußten angesichts des thematischen Umfangs oft bleiben, Abweichungen geschahen immer wider besseren Wissens, für die echten Irrtümer bin ich natürlich selbst verantwortlich und bedanke mich für eine Belehrung. 